\title{
MEJORA DEL LEAD TIMEY PRODUCTIVIDAD \\ EN EL PROCESO ARMADO DE PIZZAS \\ APLICANDO HERRAMIENTAS DE LEAN \\ MANUFACTURING
}

BRUCE ESCUDERO-SANTIAGO*

https://orcid.org/0000-0002-8693-5952

Universidad de Lima, Perú

Recibido: 29 de febrero del 2020 / Aprobado: 24 de julio del 2020

doi: https://doi.org/10.26439/ing.ind2020.n039.4915

RESUMEN: El objetivo del presente trabajo es mejorar los niveles de lead time y productividad en el proceso armado de pizzas en una mediana empresa, utilizando herramientas de lean manufacturing para implementar un método de trabajo que elimine los desperdicios o actividades que no agregaban valor al proceso. Los resultados mostraron una reducción del lead time del proceso en $99 \%$ y un incremento de la productividad hasta en $20 \%$ respecto de la situación inicial. Se constató que las herramientas implementadas, como la gráfica de equilibrio, las $5 \mathrm{~S}$ o la manufactura celular permitieron eliminar los desperdicios encontrados en el proceso.

Palabras clave: lean manufacturing / lead time / productividad

\section{LEAD TIME AND PRODUCTIVITY IMPROVEMENT IN A PIZZA ASSEMBLY PROCESS USING LEAN MANUFACTURING TOOLS}

ABSTRACT: The present work aims to improve the lead time and productivity in the pizza assembly process of a medium-sized company. To this end, lean manufacturing tools were used for implementing a work method that eliminates wastes or activities that add no value to the process. The results showed a reduction in the process lead time by $99 \%$ and an increase in the productivity of up to $20 \%$ compared to the initial situation. It was concluded that the tools used in the research, such as the line balancing graph, $5 \mathrm{~S}$ or cellular manufacturing, allowed the elimination of wastes found during the process.

Keywords: lean manufacturing / lead time / productivity

*Correo electrónico: escuderobruce@gmail.com, bruce@aloe.ulima.edu.pe 


\section{INTRODUCCIÓN}

El término manufactura esbelta (lean manufacturing) fue introducido en occidente por primera vez en 1990 con la publicación del libro La máquina que cambió al mundo, de James P. Womack (Womack y otros, 1990), basado en el estudio del sistema de producción Toyota realizado por el MIT. El autor mostraba cómo la administración, los trabajadores de línea y los proveedores podían trabajar conjuntamente en las plantas de manufactura para mejorar la eficiencia operacional a través de mejoras de la calidad y de las entregas a tiempo, reduciendo los costos, aumentando la velocidad de entrega y tiempos de ciclo, incrementando la productividad e impactando positivamente sobre los resultados financieros (Cooper et al., 2008; Monge, Cruz y López, 2013). Taiichi Ohno, diseñador del sistema de producción Toyota, fue la primera persona en reconocer la enorme cantidad de muda ${ }^{1}$ o desperdicio que existía en el gemba ${ }^{2}$ y la clasificó en siete categorías: muda de sobreproducción, muda de inventario, muda de reparaciones/rechazo de productos defectuosos, muda de movimiento, muda de sobre procesamiento, muda de espera y muda de transporte (Imai, 1998).

El principio fundamental de lean manufacturing es que el producto o servicio debe ajustarse a lo que el cliente requiere y para satisfacer estas condiciones propugna la eliminación de desperdicios. En general, las tareas que contribuyen a incrementar el valor del producto no superan el $1 \%$ del total del proceso productivo, es decir, el $99 \%$ de las actividades restantes no aportan valor y entonces constituyen desperdicios (Rajadell y Sánchez, 2010).

Existen tres fases para implementar lean manufacturing, según Tapping y Shuker (2003): conocer la demanda del cliente, establecer un flujo continuo y nivelar la producción. Se debe establecer un flujo continuo para asegurar que las unidades de trabajo correctas lleguen en el momento correcto ( $p .50$ ) teniendo como uno de sus soportes al método de inventario FIFO, el cual asegura que el trabajo más antiguo debe ser el primero en ser procesado (p. 104). Para Rother y Shook (1999), los sistemas de "flujo jalado" (PULL) son una manera eficaz de controlar la producción entre procesos que no se pueden unir en un flujo continuo (p. 48).

Según Socconini (2017), los diagramas de flujo de valor (VSM) permiten la detección de las actividades que no agregan valor al proceso (p. 103); además, considera que las $5 S$ debe representar el inicio de un sistema de mejora (p. 147); así también, según Bicheno y Holweg (2009), la manufactura celular permite reducir el lead time, reduce inventarios y permite identificar tempranamente problemas de calidad (p. 123).

1 Hace referencia a cualquier actividad que no agregue valor.

2 Palabra japonesa que significa "lugar real"; ahora, adaptada en la terminología gerencial se refiere al lugar de trabajo. 
Luego de implementar una mejora, es importante preservar los resultados; por ello, según la Secretaría Central de ISO (2015), el conocimiento, los resultados de una mejora en el proceso de una empresa deben mantenerse y poner a disposición (p. 7).

La revisión de literatura ha brindado un panorama del estado de investigación: estudios que consolidan lo realizado como alternativa de mejora, Martínez et al. (2015) en el estudio Mejora en el tiempo de atención al paciente en una Unidad de Urgencias mediante la aplicación de demostraron que, aplicando las herramientas de lean manufacturing VSM y diagrama de Spaghetti, obtuvieron una reducción promedio de tiempos de ciclo del 54 \%. Salazar (2017), en su trabajo Mejora en la productividad durante la fabricación de cabina cerrada implementando lean manufacturing en una empresa privada metalmecánica demostraría que la eliminación de actividades innecesarias incrementa la productividad en $25 \%$ y reduce los tiempos del proceso en $32 \%$ aplicando herramientas de lean manufacturing como $5 \mathrm{~S}$ y flujo continuo en la distribución de planta. Por su parte, Ríos (2018) indicó que, aplicando 5S, balance de línea y flujo continuo en la redistribución de planta, se consigue aumentar la productividad en $44,4 \%$, reducir el tiempo de producción en $24,4 \%$ y reducir las distancias recorridas de producción en $18,4 \%$.

La publicación de la Cámara de Comercio de Lima (2018) señala que, en el Perú, el $99,8 \%$ de las empresas son micro, pequeñas y medianas, que solo generan el $20 \%$ de las ventas totales del país por lo que requieren mejorar su competitividad y productividad.

Se realiza el presente estudio en una mediana empresa constituida en el año 2015 dedicada a la elaboración de productos de panadería y alimentos preparados perecederos. Sus pizzas de tamaño familiar y personal son uno de los productos con mayor volumen de producción, con tendencia al incremento de pedidos. Uno de los problemas principales que tiene el área de armado de pizzas es el incorrecto método de producción, la ineficiente distribución de las actividades y un uso ineficaz de insumos y materiales, lo que impacta negativamente en los tiempos de producción y la productividad operativa. El objetivo central es mejorar el lead time y la productividad en el proceso armado de pizzas, por lo que el objeto del presente trabajo la eliminación de los desperdicios o actividades que no agregan valor.

El aporte de este estudio queda demostrado al obtenerse mejoras en el lead time y la productividad, estableciendo un flujo continuo del producto a elaborar, con inventarios mínimos y controlados, reemplazando el exceso de sobreproducción, inventarios y tiempos de espera de la situación inicial. Como aporte social, se logró que los operarios realicen sus actividades de manera más eficiente, dejando a la empresa la posibilidad de realizar contrataciones de operarios según los requerimientos de las células de producción. 


\section{METODOLOGÍA}

La investigación es aplicada en el área de trabajo, realizando observaciones de campo ${ }^{3}$ al sistema de producción para recoger información, analizarla e implementar la mejora del proceso aplicando las herramientas de lean manufacturing. De la muestra realizada en un tiempo de cuatro semanas se recogen veintiséis valores para el lead time, la productividad de mano de obra, tiempos de espera entre las actividades del proceso en estudio e inventarios en proceso.

La investigación se ha estructurado considerando la descripción del proceso, la identificación de las actividades que no agregan valor en el proceso, la implementación de lean manufacturing a través de las herramientas: gráfica de equilibrio, las $5 \mathrm{~S}$, manufactura celular y VSM.

Según Tapping y Shuker (2003), existen tres fases para implementar lean manufacturing siguiendo este orden: comprender y satisfacer la demanda del cliente, establecer un flujo continuo para garantizar que el producto correcto llegue en el momento correcto y realizar la nivelación de la producción para distribuir el trabajo de manera uniforme (p. 50).

\subsection{Variables}

A continuación, se muestran las variables independientes (desperdicios asociados, como sobreproducción, inventarios, movimientos innecesarios, transporte de materiales, tiempo de espera, defectos) y las variables dependientes (lead time y productividad). Los desperdicios asociados son los mismos para ambas variables dependientes.

\section{Y1: Lead time}

X1: Sobreproducción, inventarios, movimientos innecesarios, transporte de materiales, tiempo de espera, defectos.

Y2: Productividad (unidades $/ \mathrm{h}-\mathrm{h}^{4}$ )

X2: Sobreproducción, inventarios, movimientos innecesarios, transporte de materiales, tiempo de espera, defectos.

\section{RESULTADOS - SITUACIÓN INICIAL}

La demanda diaria de pizzas es cambiante según el requerimiento del cliente. El proceso en estudio comienza desde la colocación de stickers de fecha de vencimiento

3 Estar en el lugar donde ocurren los hechos.

4 Horas-hombre. 
en los ingredientes hasta colocar la pizza envuelta en papel film en la jaba, el detalle se describe en el $\operatorname{SIPOC}^{5}$ (tabla 1). El producto final se distribuye a las tiendas en diferentes distritos de Lima.

Tabla 1

SIPOC

\begin{tabular}{|c|c|c|c|c|c|c|c|c|c|}
\hline \multirow[b]{2}{*}{ PROVEEDOR } & \multicolumn{5}{|c|}{ ENTRADAS } & \multirow[b]{2}{*}{ PROCESO } & \multirow[b]{2}{*}{ SALIDAS } & \multirow{2}{*}{\begin{tabular}{|c|} 
REQUERIMIENTOS \\
DE LAS \\
SALIDAS \\
\end{tabular}} & \multirow[b]{2}{*}{ CLIENTE } \\
\hline & $\begin{array}{l}\text { Mano de } \\
\text { obra }\end{array}$ & Material & Máquina & Cantidad & Descripción & & & & \\
\hline $\begin{array}{l}\text { Gerencia } \\
\text { general }\end{array}$ & Secretaria & & & 1 & $\begin{array}{c}\text { Con experiencia de } \\
3 \text { años en labores } \\
\text { administrativas }\end{array}$ & \multirow{4}{*}{$\begin{array}{c}\text { Colocar sticker } \\
\text { de } \\
\text { ingredientes/ } \\
\text { fecha de } \\
\text { vencimiento en } \\
\text { plato }\end{array}$} & \multirow{4}{*}{$\begin{array}{c}\text { Platos con } \\
\text { stickers } \\
\text { adheridos }\end{array}$} & \multirow{4}{*}{$\begin{array}{c}\text { Platos con stickers } \\
\text { adheridos }\end{array}$} & \multirow{4}{*}{$\begin{array}{l}\text { Área de } \\
\text { producción }\end{array}$} \\
\hline Almacén & & & $\begin{array}{c}\text { Fechador } \\
\text { manual }\end{array}$ & 1 & \begin{tabular}{|c|} 
Equipo portátil \\
pequeño para \\
adherir sticker de \\
fechas de \\
vencimiento \\
\end{tabular} & & & & \\
\hline Almacén & & $\begin{array}{l}\text { Platos de } \\
\text { tecnopor }\end{array}$ & & $\begin{array}{c}\text { Según } \\
\text { volumen de } \\
\text { producción }\end{array}$ & \begin{tabular}{|c|} 
Platos de \\
poliestireno \\
expandido, material \\
plástico espumado
\end{tabular} & & & & \\
\hline Almacén & & $\begin{array}{c}\text { Stickers } \\
\text { autoadhesivos }\end{array}$ & & $\begin{array}{c}\text { Según } \\
\text { volumen de } \\
\text { producción }\end{array}$ & & & & & \\
\hline $\begin{array}{l}\text { Gerencia } \\
\text { general }\end{array}$ & \begin{tabular}{|c} 
Operario(s) \\
de \\
producción
\end{tabular} & & & $5-9$ & $\begin{array}{l}\text { Con / sin } \\
\text { experiencia }\end{array}$ & \multirow{2}{*}{$\begin{array}{l}\text { Colocar plato } \\
\text { en mesa }\end{array}$} & \multirow{12}{*}{$\begin{array}{c}\text { Producto } \\
\text { terminado } \\
\text { que } \\
\text { recibirá } \\
\text { cliente }\end{array}$} & \multirow{12}{*}{$\begin{array}{c}\text { Producto terminado } \\
\text { con stickers legibles, } \\
\text { film que envuelve el } \\
\text { producto sin roturas }\end{array}$} & \multirow{12}{*}{$\begin{array}{c}\text { Área de } \\
\text { despacho }\end{array}$} \\
\hline Almacén & & $\begin{array}{l}\text { Platos con } \\
\text { stickers } \\
\text { adheridos }\end{array}$ & & \begin{tabular}{|c|} 
Según \\
volumen de \\
producción
\end{tabular} & & & & & \\
\hline $\begin{array}{l}\text { Área de } \\
\text { horneado }\end{array}$ & & $\begin{array}{c}\text { Masa } \\
\text { horneada }\end{array}$ & & $\begin{array}{c}\text { Según } \\
\text { volumen de } \\
\text { producción }\end{array}$ & $\begin{array}{r}\text { Bajo en dorado / } \\
\text { tamaño definido } \\
\text { para pizza tamaño } \\
\text { familiar y personal }\end{array}$ & $\begin{array}{c}\text { Colocar masa } \\
\text { horneada en } \\
\text { plato }\end{array}$ & & & \\
\hline Almacén & & Sal & & $\begin{array}{c}\text { Según } \\
\text { volumen de }\end{array}$ & $\begin{array}{c}\text { Bolsas herméticas } \\
\text { de } 5 \mathrm{~kg} \text {, en buen }\end{array}$ & \begin{tabular}{|c|}
$\begin{array}{c}\text { Colocar salsa } \\
\text { de tomate en } \\
\text { masa horneada }\end{array}$ \\
\end{tabular} & & & \\
\hline & & de tomate & & producción & estado & $\begin{array}{c}\text { Expandir salsa } \\
\text { de tomate }\end{array}$ & & & \\
\hline & & & & Según & \begin{tabular}{|} 
Queso rallado en \\
bolsas herméticas
\end{tabular} & $\begin{array}{r}\text { Colocar queso } \\
\text { rallado sobre } \\
\text { salsa de tomate }\end{array}$ & & & \\
\hline | Almacen & & Queso & & $\begin{array}{l}\text { volumen de } \\
\text { producción }\end{array}$ & \begin{tabular}{|c|} 
con atmostera \\
modificada de 3 \\
$\mathrm{~kg}$, en buen estado
\end{tabular} & $\begin{array}{c}\text { Expandir queso } \\
\text { rallado sobre } \\
\text { salsa de tomate }\end{array}$ & & & \\
\hline Almacén & & Jamonada & & \begin{tabular}{|l} 
Según \\
volumen de \\
producción
\end{tabular} & \begin{tabular}{|c|} 
Cortada en \\
cuadrados, en \\
bolsas al vacío de \\
$1 \mathrm{~kg}$, en buen estado
\end{tabular} & $\begin{array}{c}\text { Colocar } \\
\text { jamonada sobre } \\
\text { queso rallado }\end{array}$ & & & \\
\hline $\begin{array}{l}\text { Área de } \\
\text { producción }\end{array}$ & & & Bitafiladora & 2 & $\begin{array}{l}\text { Soporte para } \\
\text { papel film }\end{array}$ & \multirow{2}{*}{$\begin{array}{c}\text { Bitafilar plato } \\
\text { con masa } \\
\text { horneada, salsa } \\
\text { de tomate, } \\
\text { queso rallado, } \\
\text { jamonada }\end{array}$} & & & \\
\hline $\begin{array}{l}\text { Área de } \\
\text { producción }\end{array}$ & & $\begin{array}{l}\text { Producto a } \\
\text { envolver }\end{array}$ & & $\begin{array}{c}\text { Según } \\
\text { volumen de } \\
\text { producción }\end{array}$ & $\begin{array}{c}\text { Plato con masa } \\
\text { horneada, salsa } \\
\text { tomate, queso } \\
\text { rallado, jamonada }\end{array}$ & & & & \\
\hline Almacén & & Stickers & & \begin{tabular}{|c|} 
Según \\
volumen de \\
producción
\end{tabular} & $\begin{array}{r}\text { Stickers con logo } \\
\text { de la empresa }\end{array}$ & $\begin{array}{c}\text { Colocar sticker } \\
\text { de marca de } \\
\text { cliente }\end{array}$ & & & \\
\hline $\begin{array}{l}\text { Área de } \\
\text { despacho }\end{array}$ & & Jabas & & $\begin{array}{l}\text { Según } \\
\text { volumen de } \\
\text { producción }\end{array}$ & & $\begin{array}{l}\text { Colocar } \\
\text { producto } \\
\text { bitafilado en } \\
\text { jaba }\end{array}$ & & & \\
\hline
\end{tabular}

Elaboración propia

5 Herramienta utilizada para analizar el proceso y su entorno. 


\subsection{Desperdicios identificados en el proceso}

La información recogida mostró que los mayores valores de tiempos de espera alcanzaban un $72,5 \%$, que correspondían a las actividades "colocar stickers de fecha de vencimiento-ingredientes en el plato" y "colocar plato en mesa", teniendo como causa la sobreproducción en colocar los stickers, seguida por el tiempo de espera entre "expandir queso rallado" y "colocar jamonada" con un 7,5 \%.

Se evidenció que el lead time podía llegar a 6,9 horas para producir "un" producto y que las horas-hombre extras podían tener un promedio de 9,30 horas por los tiempos empleados en realizar actividades que no agregaban valor, ocasionando un impacto negativo en los niveles de productividad.

Los desperdicios identificados en el proceso, como sobreproducción, inventarios de stickers de fecha de vencimiento e ingredientes en platos, movimientos innecesarios, transporte en recipiente de la salsa de tomate que se agregará sobre la masa horneada, tiempos de espera, defectos, entre otros, se muestran en el VSM actual.

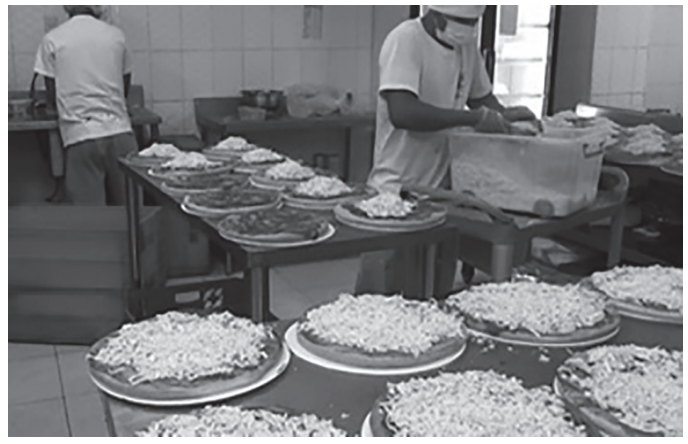

Figura 1. Desperdicios identificados

Elaboración propia

En la figura 2 se observa el VSM actual del proceso armado de una pizza de tamaño familiar para elaborar un solo producto, el cual incluye los desperdicios identificados: tiempos de espera entre cada actividad, tiempos de ciclo6, inventarios en proceso entre cada actividad y valor del lead time. La manera de producir era la misma para las pizzas tamaño familiar y tamaño personal, por ello, solo se representa el VSM actual para la pizza tamaño familiar. El lead time lo conforman los tiempos de espera ${ }^{7}$ (tiempos de valor no agregado) más los tiempos de ciclo ${ }^{8}$ (tiempo de valor agregado).

\footnotetext{
6 Valores promedio.

7 Tiempo que una unidad de trabajo esperará a que una actividad o proceso posterior esté listo para trabajar en él.

8 Tiempo que transcurre desde el comienzo de una actividad o proceso individual hasta que se completa.
} 


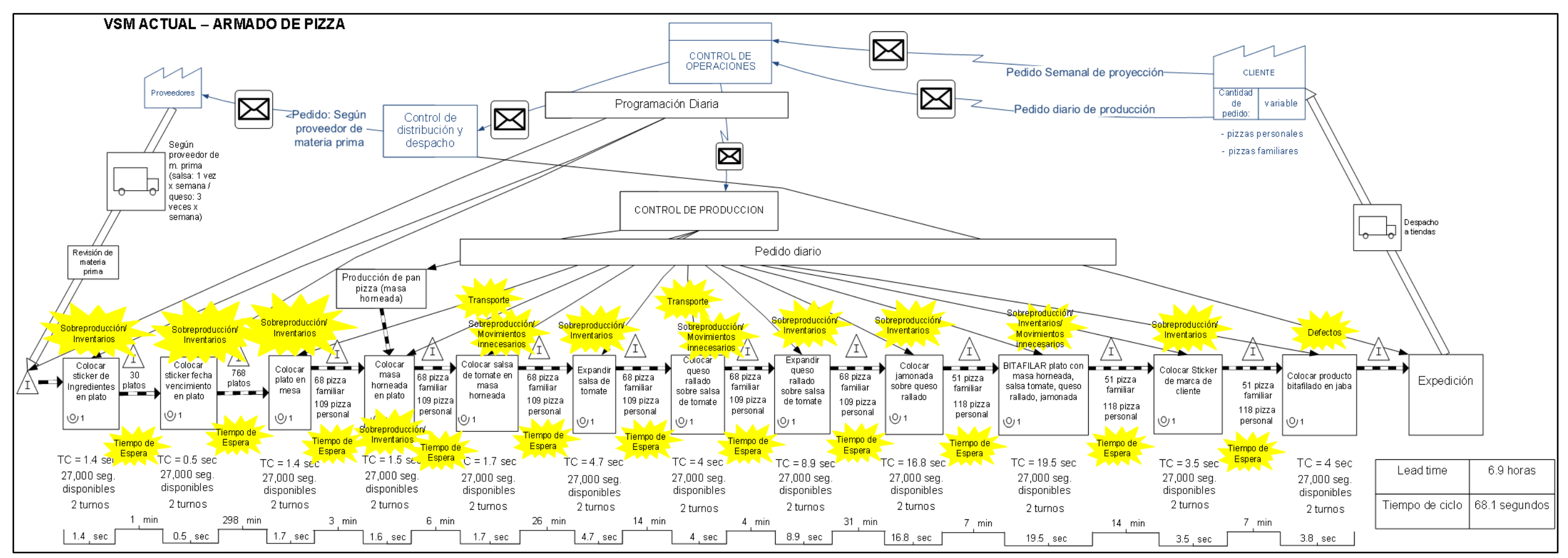

Figura 2. VSM actual - armado de pizza

Elaboración propia 

Para la fase "demanda del cliente", se definió realizar la prueba piloto para pizzas tamaño familiar, considerándose el tiempo de ciclo total (tabla 2), que incluye, según Niebel (1990), el $4 \%$ como factor de tolerancia básica por fatiga y el $2 \%$ de factor de tolerancia por estar de pie, basado en información de la Oficina Internacional del Trabajo (p. 414).

Tabla 2

Tiempos de ciclo proceso "armado de pizzas" - situación inicial

\begin{tabular}{llcc}
\hline Código & \multicolumn{1}{c}{ Actividad } & $\begin{array}{c}\text { Pizza familiar } \\
\text { Valor promedio } \\
\text { (segundos) }\end{array}$ & $\begin{array}{c}\text { Pizza personal } \\
\text { Valor promedio } \\
\text { (segundos) }\end{array}$ \\
\hline A & $\begin{array}{l}\text { Colocar stickers - ingredientes, } \\
\text { fecha de vencimiento }\end{array}$ & 1,9 & 1,9 \\
B & Colocar plato en mesa & 1,7 & 1,9 \\
C & Colocar masa horneada en plato & 1,6 & 1,2 \\
D & Colocar salsa de tomate & 1,7 & 1,0 \\
E & Exparcir salsa de tomate & 4,7 & 4,0 \\
F & Colocar queso rallado & 4,0 & 3,7 \\
G & Espandir queso rallado & 8,9 & 1,9 \\
H & Colocar jamonada & 16,8 & 4,9 \\
I & Bitafilar & 19,5 & 10,4 \\
J & Colocar sticker (marca) & 3,5 & 1,8 \\
K & Colocar producto en jaba & 3,8 & 4,0 \\
Tiempo total de ciclo & 68,1 & 36,7 \\
\hline
\end{tabular}

Elaboración propia

El turno de 9,5 horas incluye una hora para la limpieza del área de producción (media hora antes de iniciar producción y media hora luego de terminar la producción), así como una hora de refrigerio, por lo tanto, para el cálculo del tiempo takt se consideran 7,5 horas de tiempo disponible, lo cual representa 27000 segundos.

Considerando un turno en la producción de armado de pizzas tamaño familiar, y con los valores del tiempo disponible por día y una demanda promedio por día de setecientas unidades, se estableció el tiempo takt y el número de operarios requeridos (tabla 3 ).

$$
\text { tiempo }=\frac{\text { tiempo disponible }}{\text { demanda }}=\frac{27000 \mathrm{seg}}{700 \text { unds. }}=38,6 \frac{\mathrm{seg}}{\mathrm{und} .}
$$




$$
\text { Número de operarios }=\frac{\text { tiempo de ciclo total }}{\text { tiempo }}=\frac{68,1 \mathrm{seg}}{38,6 \mathrm{seg}}=2
$$

Tabla 3

Especificaciones para establecer el flujo continuo - pizza tamaño familiar

\begin{tabular}{ccccc}
\hline $\begin{array}{c}\text { Tiempo de ciclo } \\
\text { total (seg) }\end{array}$ & $\begin{array}{c}\text { Tiempo disponible } \\
(\mathrm{seg})\end{array}$ & $\begin{array}{c}\text { Demanda } \\
\text { (producción) }\end{array}$ & $\begin{array}{c}\text { Tiempo takt } \\
\text { (seg/und.) }\end{array}$ & $\begin{array}{c}\text { Número de } \\
\text { operarios }\end{array}$ \\
\hline 68,1 & 27000 & 700 & 38,6 & 2 \\
\hline
\end{tabular}

Elaboración propia

Luego, en la fase de flujo continuo, se definió la distribución de actividades para cada operario, elaborándose el diseño la gráfica de equilibrio con dos operarios para pizza tamaño familiar (tabla 4). Se observa que, para la actividad "A" (colocar stickers) se multiplica el tiempo de ciclo por 5 para acercarse al tiempo takt y alcanzar un flujo continuo de trabajo, lo cual significa que el operario 1 debe realizar cinco veces la actividad de colocar stickers a los platos/bandejas antes de realizar la siguiente actividad.

Tabla 4

Diseño de gráfica de equilibrio con 2 operarios - pizza tamaño familiar

\begin{tabular}{cccc}
\hline $\begin{array}{c}\text { Tiempos } \\
(\mathrm{seg})\end{array}$ & $\begin{array}{c}\text { Tiempo de ciclo } \\
(\mathrm{seg})\end{array}$ & $\begin{array}{c}\text { Takt time } \\
(\mathrm{seg})\end{array}$ & $\begin{array}{c}\text { Diferencia } \\
(\mathrm{seg})\end{array}$ \\
\hline $5 \times 1,9+19,5+3,5+4.0$ & 36,4 & 38,6 & 2,2 \\
$1,7+1,6+1,7+4,7+4,0+8,9+16,8$ & 39,4 & 38,6 & $-0,8$ \\
\hline
\end{tabular}

Elaboración propia 


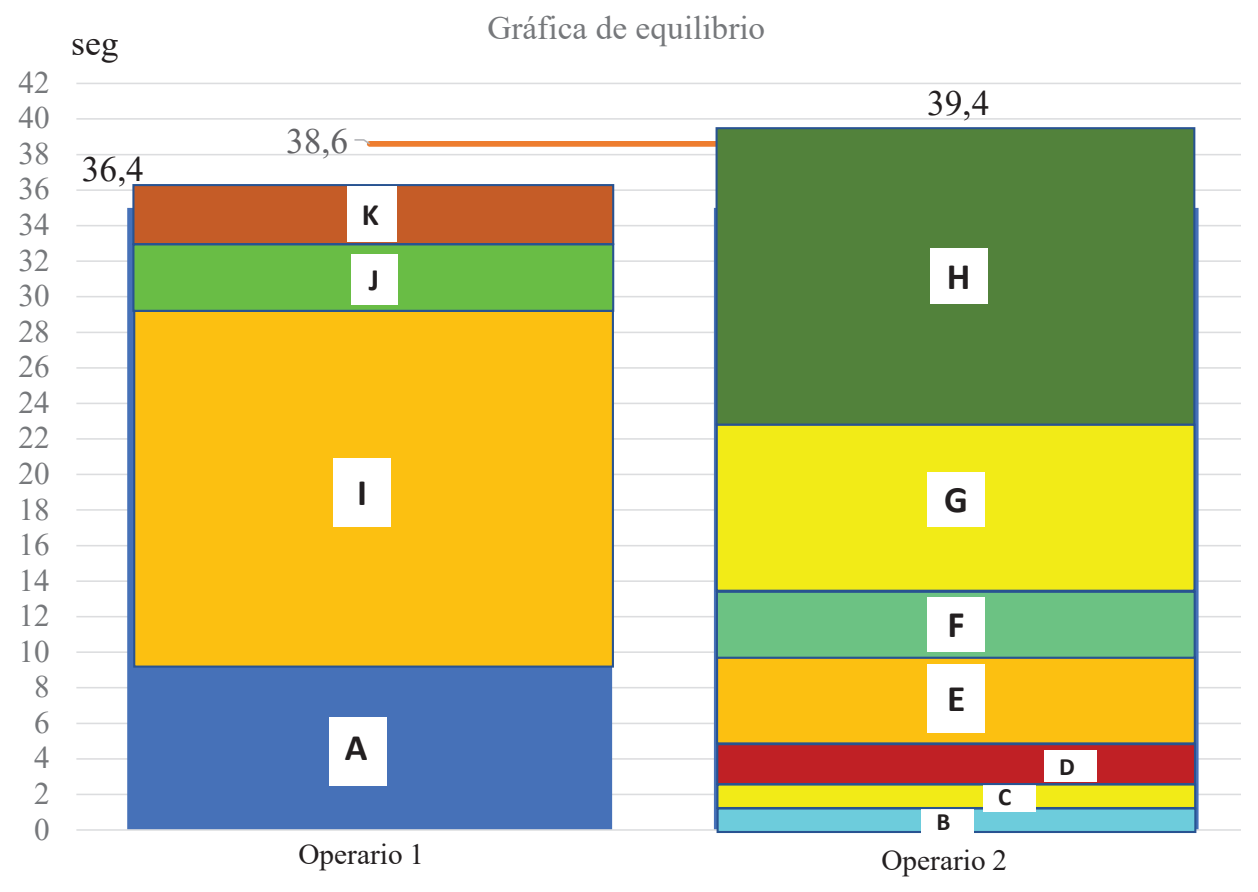

Figura 3. Gráfica de equilibrio con dos operarios - pizza tamaño familiar

Elaboración propia

En la tabla 4 se observa que se sobrepasa el tiempo takt en 0,8 segundos para las actividades del operario 2, por lo que decidió aceptarse, ya que representaba la mejor distribución posible de actividades. La diferencia entre las actividades asignadas al operario 1 y al operario 2 es que las del operario 2 involucran la manipulación de los alimentos que contiene la pizza (colocar salsa de tomate, queso rallado, jamonada), mientras que las actividades del operario 1 involucran colocar stickers, envolver la pizza y colocarla en la jaba.

Con la información de la figura 3 se elaboró el diseño de célula para dos operarios - pizza tamaño familiar (figura 4). 


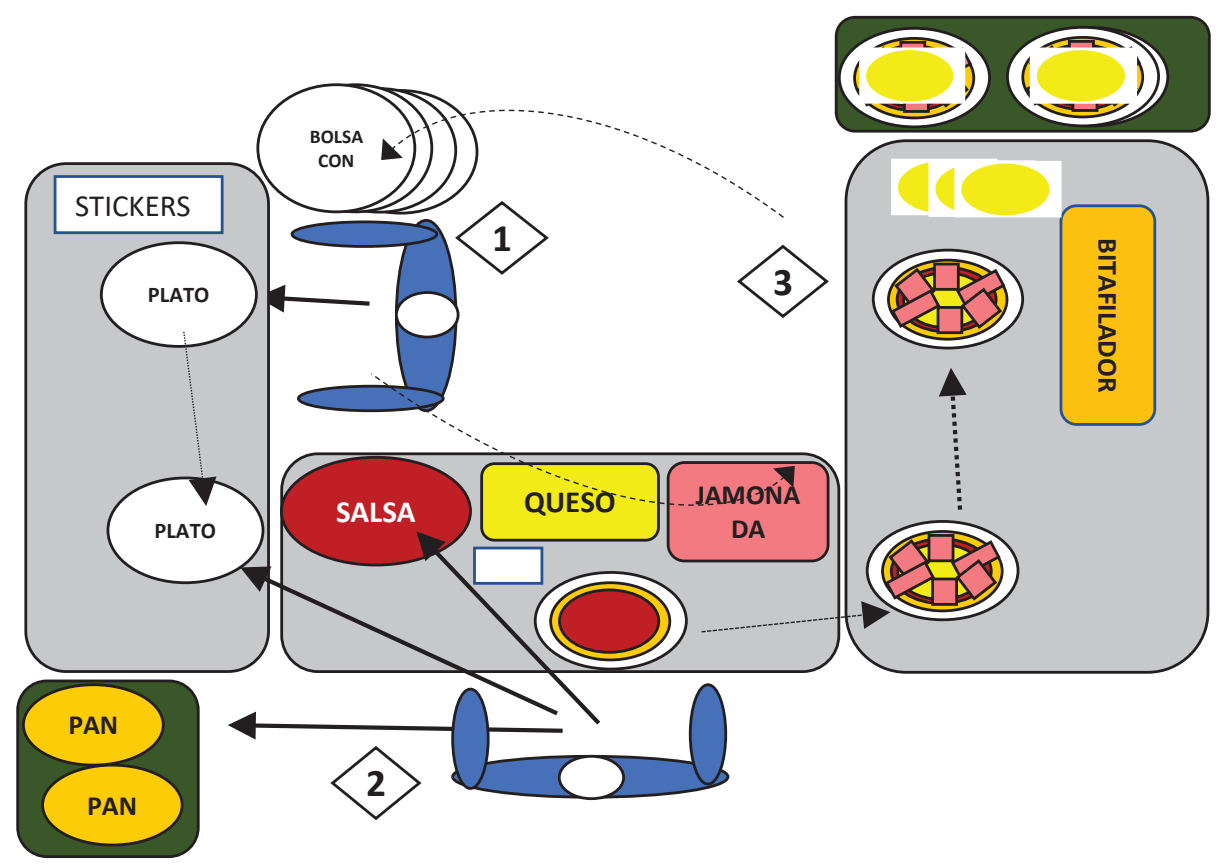

Figura 4. Diseño de célula para 2 operarios - pizza tamaño familiar

Elaboración propia

Luego, se aplican las 5S. Se empezó seleccionando los insumos necesarios para realizar la producción; luego, se definió en las mesas de trabajo la ubicación de los materiales e insumos que los operarios necesitaban para la realización de las actividades asignadas a cada uno de ellos (figuras 5 y 6). La limpieza es una actividad que se tiene implementada en la empresa como parte de la certificación en BPM. El tiempo otorgado a dicha actividad fue de treinta minutos antes de iniciar el proceso armado de pizzas y treinta minutos después de culminada la producción a realizar. Definida la ubicación de los materiales e insumos en las mesas de trabajo, se estandariza el nuevo método de trabajo a través de videos; posteriormente, se estableció formato de evaluaciones periódicas para medir el nivel de cumplimiento de los estándares establecidos. 


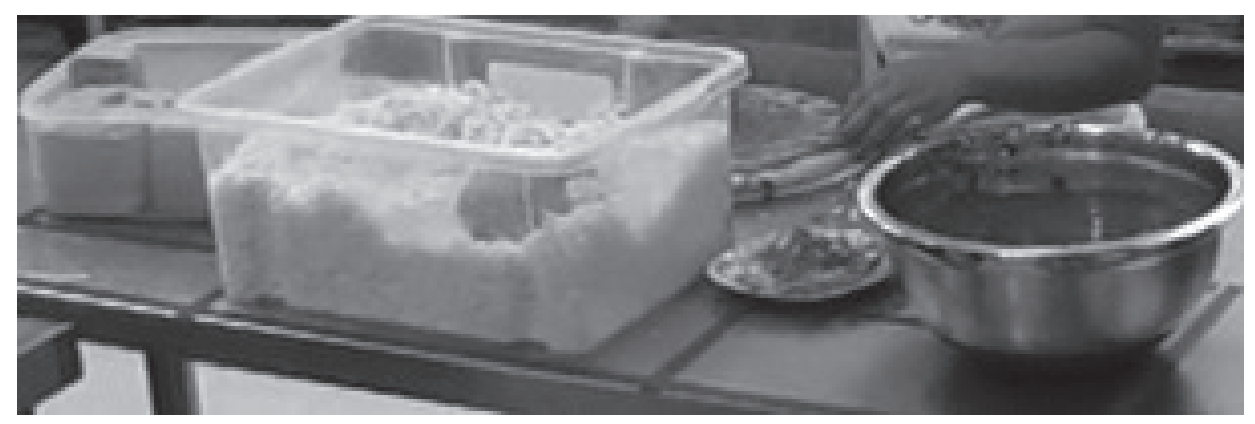

Figura 5. Disposición de insumos en área de trabajo

Elaboración propia

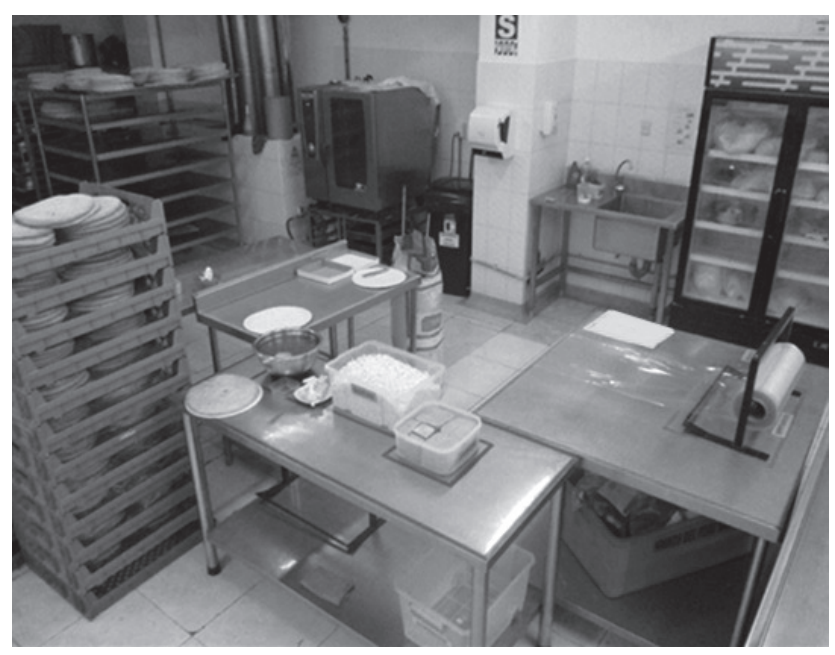

Figura 6. Disposición de mesas - vista frontal

\section{Elaboración propia}

A continuación, se observa el VSM futuro, que presenta un sistema jalar (en lugar del sistema de empuje que se grafica en el VSM actual). Esta gráfica representa el plan de inicio para la construcción del nuevo esquema de trabajo, que incluye: flujo de movimiento de pieza única (one piece flow), implementación del método de inventario FIFO

9 Significado de "primero en entrar, primero en salir". 
(First In-First Out), un método de control de trabajo utilizado para asegurar que el trabajo más antiguo (primero en entrar) sea el primero en ser procesado (primero en salir).

Luego de la prueba piloto, se procedió a diseñar la gráfica de equilibrio, manufactura celular, $5 \mathrm{~S}$ para el armado de pizza tamaño personal con dos y tres operarios y pizza tamaño familiar con tres operarios.

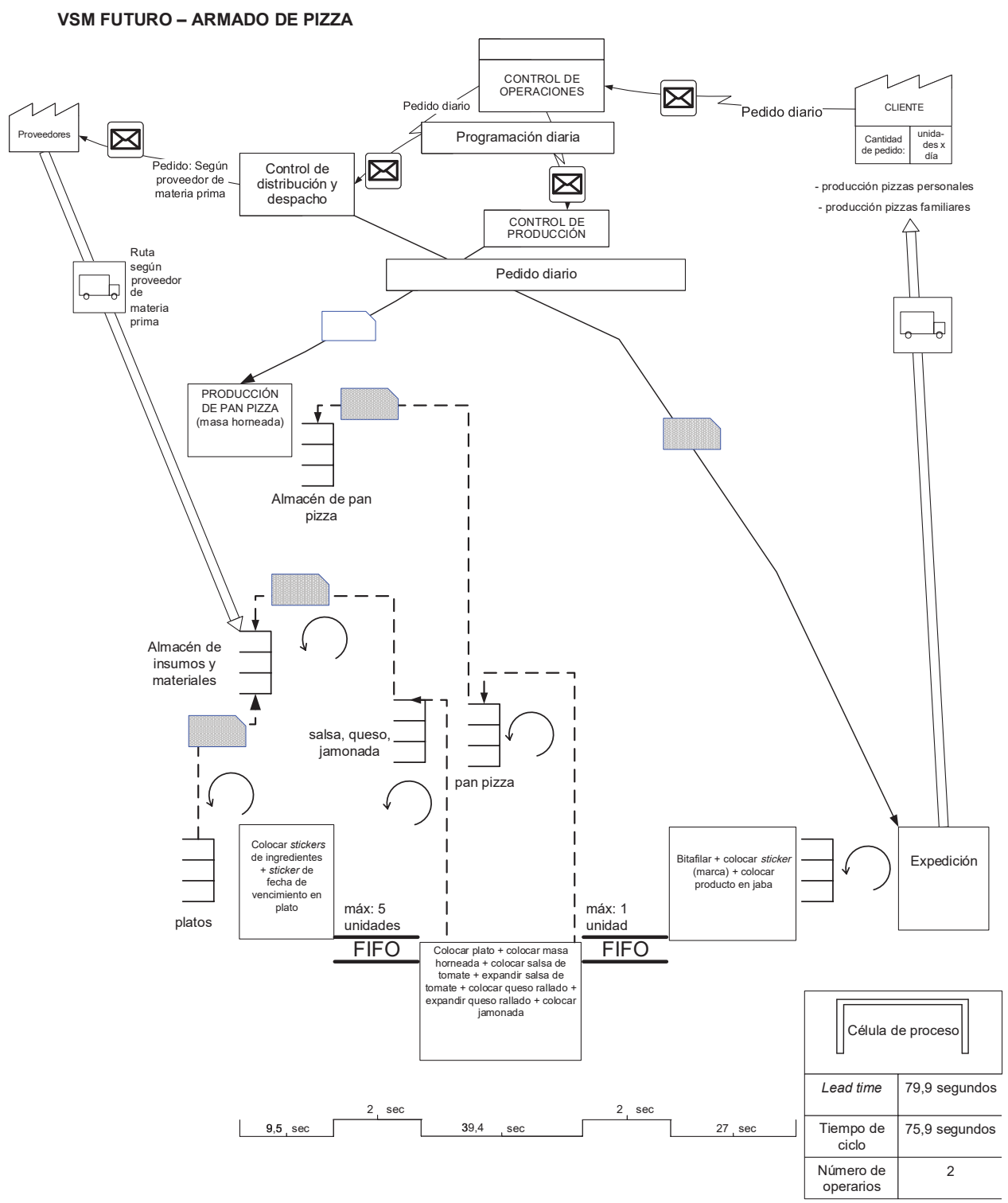

Figura 7. VSM futuro

Elaboración propia 


\section{RESULTADOS - SITUACIÓN POSTERIOR}

En la prueba piloto se observó que, para producir una unidad, el operario 1 termina más pronto que el operario 2 en realizar la secuencia de actividades asignadas según la gráfica de equilibrio, lo que le permite realizar actividades complementarias como:

- Mover el queso que se encuentra en el recipiente mientras el operario 2 coloca la jamonada.

- Limpiar la mesa del área de trabajo en donde el queso ha caído del plato.

- $\quad$ Traer jamonada del refrigerador hacia el área de trabajo de operario 2.

- Limpiar jaba.

Se estableció que, cada vez que el operario 1 observara que una pizza había llegado a su área de trabajo, tenía que detener alguna de las actividades complementarias que estaba realizando para hacer las que estaban asignadas en el diseño de célula.

Se observó, en promedio, que de cada diez masas horneadas una no tenía las medidas correctas, así que se empleaba tiempo en revisar que la masa horneada que fuera a elegir tuviera las medidas correctas. Con el transcurrir del tiempo, la cantidad de producción disminuye debido a las siguientes razones:

- Tamaño incorrecto de masa horneada (pan pizza), lo que hacía necesario cortar los bordes del pan hasta que alcanzara el tamaño correcto.

- Cansancio en los operarios por realizar el trabajo de pie.

En la situación inicial, se encontró un número variable de pasos que realizaban los operarios para realizar las actividades del proceso mientras, que en la situación posterior el número de pasos disminuye (tabla 5). Asimismo, en la figura 9 se observa una disminución en la distancia recorrida para realizar la producción respecto de la situación inicial, que es el resultado de implementar un método de producción de flujo continuo.

\section{Tabla 5}

Número de pasos en layout

\begin{tabular}{lll}
\hline & Situación Inicial & Situación posterior \\
\hline $\begin{array}{l}\text { Número de } \\
\text { pasos }\end{array}$ & Variable & Con 2 operarios: 12 \\
\hline
\end{tabular}

Elaboración propia 


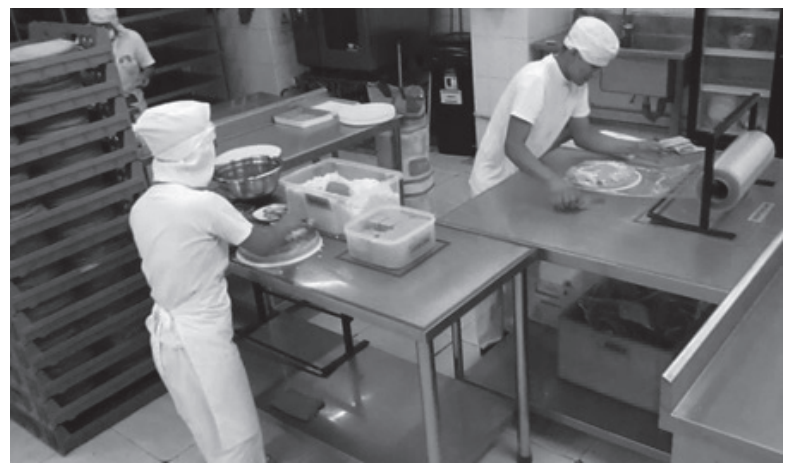

Figura 8. Célula de producción

Elaboración propia

Al realizar una comparación entre la situación inicial con la situación posterior (tabla 6), se observan las mejoras alcanzadas para iguales o similares niveles de producción totales de pizza (tamaño familiar más tamaño personal). Por ejemplo, para una producción total de 1690 unidades, se obtuvo una productividad de 35,4 unidades/h-h con seis operarios, es decir, antes se requerían siete operarios (un operario adicional provenía de otro proceso para realizar horas extras y culminar la producción); luego, con la mejora implementada, se requieren seis operarios, lo que representa una reducción de $66 \%$ en las horas de trabajo. En la tabla 6 se muestra la reducción en los costos de horas extras para producciones iguales o similares entre la situación inicial y la situación posterior.

Considerando el área disponible para el proceso en estudio, para un turno de trabajo y una producción máxima de 1274 unidades para pizza tamaño familiar y 1425 unidades para pizza tamaño personal, la capacidad instalada será de dos células con tres operarios cada una. 

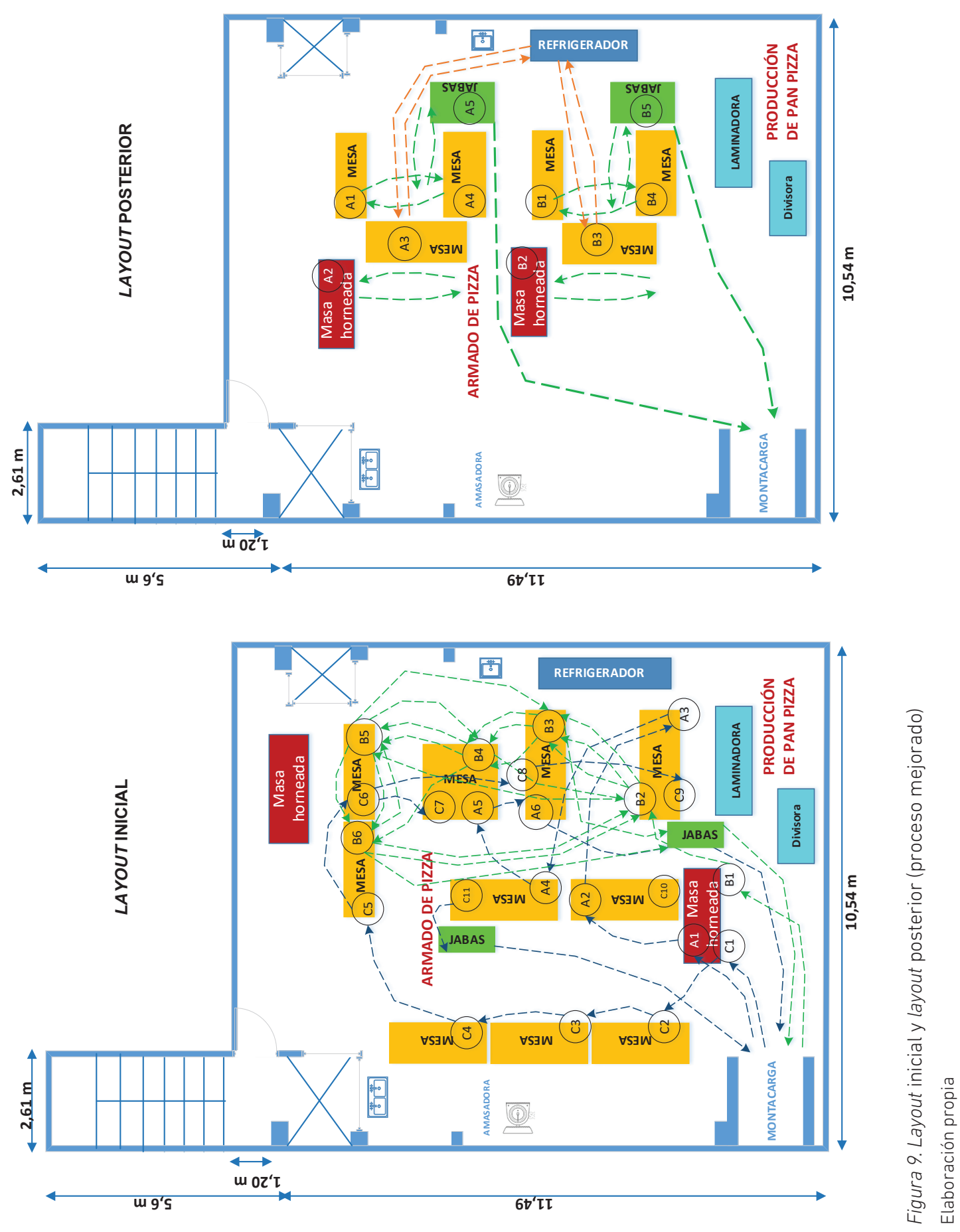
Tabla 7

Resumen: situación inicial - situación posterior

\begin{tabular}{|c|c|c|c|c|}
\hline N. ${ }^{\circ}$ & Indicador & Situación inicial & Situación posterior & Ahorro (\%) \\
\hline 1 & $\begin{array}{l}\text { Lead time } \\
\text { (producción de } \\
\text { una unidad) }\end{array}$ & 6,9 horas & 2,43 minutos & $-99,40$ \\
\hline \multirow[t]{4}{*}{2} & Productividad & 35,5 unidades con 8 operarios & 41,5 unidades con 5 operarios & 17,00 \\
\hline & & hora-hombre & hora-hombre & \\
\hline & & 32,9 unidades con 8 operarios & 39,4 unidades con 6 operarios & 20,00 \\
\hline & & hora-hombre & hora-hombre & \\
\hline 2,1 & $\begin{array}{l}\text { Tiempo de } \\
\text { valor no } \\
\text { agregado } \\
\text { (VNA) }\end{array}$ & 6,9 horas & 1,18 minutos & $-99,70$ \\
\hline 2,2 & $\begin{array}{l}\text { Tiempo de } \\
\text { valor } \\
\text { agregado (VA) }\end{array}$ & 68,1 segundos & 64,4 segundos & $-5,50$ \\
\hline \multirow[t]{2}{*}{3} & Inventario en & 68 unidades (pizza familiar) & $1-3$ unidades & $-98,50$ \\
\hline & $\begin{array}{l}\text { proceso } \\
\text { (producción } \\
\text { de una unidad) }\end{array}$ & 118 unidades (pizza personal) & & \\
\hline \multirow[t]{2}{*}{4} & horas-hombre & 23:04 & $12: 10$ & $-47,00 \%$ \\
\hline & & $27: 49: 00$ & $11: 30$ & $-59,00 \%$ \\
\hline
\end{tabular}

Nota. Los datos provienen de registros de producción y de observación en el área de trabajo. Elaboración propia

\section{DISCUSIÓN}

Con el objetivo de mejorar el lead time y la productividad operativa del proceso armado de pizzas, los resultados reflejaron la elaboración de un producto en promedio de 2,43 minutos, lo que representa una reducción del lead time en $99 \%$ y un incremento de la productividad de $17 \%$ a $20 \%$ respecto de la situación inicial. Esto quiere decir que la implementación de un flujo continuo, utilizando herramientas como la gráfica de equilibrio, 5S, VSM y manufactura celular permitió eliminar los desperdicios de sobreproducción, exceso de inventarios, movimientos innecesarios, transporte de materiales y tiempos de espera. Ordenar y clasificar los insumos necesarios en las mesas que formaron la célula (definidos en la gráfica de equilibrio y representados en el VSM) permitieron implementar uno de los conceptos principales de lean manufacturing que es el one piece flow ("flujo de una pieza"), manteniendo inventarios controlados, como por ejemplo en la colocación de stickers en los platos. 
Frente a lo mencionado, se confirma que existe relación entre las variables independientes (desperdicios) y las variables dependientes (el lead time y la productividad).

Estos resultados son similares a lo encontrado por Salazar (2017), quien concluye que al implementar herramientas de lean manufacturing como $5 \mathrm{~S}$ y establecer un flujo continuo en la distribución de planta, se eliminan actividades innecesarias y se logra incrementar la productividad en un $25 \%$. Así también, Martínez y otros (2015) concluyen que el uso de herramientas como el VSM y el diagrama de spaguetti permite una reducción promedio de tiempos de ciclo del 54 \%. Los resultados obtenidos también son similares a lo encontrado por Ríos (2018), quien concluye que al aplicar las 5S, balance de línea y establecer un flujo continuo en la redistribución de la planta de producción obtuvo un aumento de la productividad de $44,4 \%$ y una reducción del tiempo de producción en $24,4 \%$. En tal sentido, bajo lo referido anteriormente y al analizar estos resultados, confirmamos que mientras menos desperdicios existan en el proceso mejor será nivel de lead time y productividad.

Según la Secretaría Central de ISO (2015), una empresa debe determinar los conocimientos necesarios para la operación de sus procesos, debiendo mantenerse y ponerse a disposición según sea necesario. Estos conocimientos pueden ser adquiridos con la experiencia o los resultados de las mejoras en los procesos (p. 7); por ello, es importante que en la capacitación de operarios que inician labores en la empresa se muestren los tiempos de ciclo, los VSM, los diseños de célula, las gráficas de equilibrio, la ubicación de los insumos y materiales y los procedimientos visuales.

\section{CONCLUSIONES}

- Se reordenó el área de producción a través del uso de las gráficas de equilibrio, VSM, 5S y manufactura celular, lo cual permitió un mínimo de distancia recorrida de doce a catorce pasos respecto de la situación inicial, la cual era variable e indeterminada.

- Se lograron reducir los costos de horas extras para niveles de producción iguales o similares (tabla 6).

- Se demostró el incremento en la productividad con un menor número de operarios respecto de la situación inicial para iguales o similares niveles de producción (tabla 6).

- Para un incremento de la producción mayor de 1274 unidades para pizza tamaño familiar y mayor de 1425 unidades para pizza tamaño personal, se debe habilitar el turno noche teniendo en cuenta la capacidad instalada: una célula con tres operarios para el armado de pizza tamaño familiar y una célula con tres operarios para el armado de pizza tamaño personal. 
- Es necesario evaluar la relación beneficio-costo de mantener procesos realizados en la organización o elegir servicios de terceros para su realización, como es el caso del proceso "producción de masa horneada (pan pizza)".

\section{REFERENCIAS}

Bicheno, J. y Holweg, M. (2009). The Lean Toolbox the Essential Guide to Lean Transformartion [La caja de herramientas esbelta: la guía esencial para la transformación esbelta] [fourth edition]. United Kingdom: PICSIE Books.

Cámara de Comercio de Lima (10 de diciembre del 2018). Urge aumentar la competitividad de mypes. La cámara. Recuperado de https://www.camaralima.org.pe/ repositorioaps/0/0/par/r857_3/eventos\%202.pdf

Cooper, R y Maskell, B. (2008) How to manage through worse-before better [Cómo gestionar lo peor antes que lo mejor]. MIT Sloan Management Review, 49(4), 58-65.

Imai, M. (1998). Cómo implementar el kaizen en el sitio de trabajo (gemba) [traducido de la primera edición]. Colombia: McGraw-Hill Interamericana S.A.

Martínez, P.; Martínez, J.; Nuño, P. y Cavazos, J. (2015). Mejora en el tiempo de atención al paciente en una unidad de urgencias mediante la aplicación de manufactura esbelta. Revista Información tecnológica, 26(6), 187-198. Recuperado de https:// scielo.conicyt.cl/scielo.php?script=sci_arttext\&pid=S0718-07642015000600019

Monge, C.; Cruz, J. y López, F. (2013). Impacto de la manufactura esbelta, manufactura sustentable y mejora continua en la eficiencia operacional y responsabilidad ambiental en México. Revista Información tecnológica, 24(4), 15-32. Recuperado de https://scielo.conicyt.cl/scielo.php?script=sci_arttext\&pid=S0718-0764201 3000400003

Niebel, B. y Freivalds, A. (1990). Ingeniería Industrial. Métodos, tiempos y movimientos [tercera edición]. México: Alfaomega.

Rajadell, Carreras, M. y Sánchez García, J. L. (2010). Lean Manufacturing, la evidencia de una necesidad. España: Díaz de Santos.

Ríos, E. (2018). Aplicación de lean manufacturing para aumentar la productividad de la línea de producción de calzado de seguridad GYW de la empresa SEGUSA SAC [tesis de grado, Universidad Nacional de Trujillo, Perú]. Repositorio institucional de la Universidad Nacional de Trujillo. Recuperado de http://dspace.unitru.edu.pe/ bitstream/handle/UNITRU/11131/RIOS\%20BERNUY\%2c\%20Edinson\%20Eloy. pdf?sequence $=1$ \&isAllowed $=y$ 
Rother, M. y Shook, J. (1999). Observar para crear valor. Cartografía de la cadena de valor para agregar valor y eliminar "muda" (versión 1.2). USA: The Lean Enterprise Institute.

Salazar, M. (2017). Mejora en la productividad durante la fabricación de cabina cerrada implementando lean manufacturing en una empresa privada metalmecánica [tesis de grado, Universidad San Ignacio de Loyola, Perú]. Repositorio institucional de la Universidad San Ignacio de Loyola. Recuperado de http://repositorio.usil.edu. pe/bitstream/USIL/3212/1/2018_Salazar-Bozzeta.pdf

Secretaría Central de ISO (2015). Norma Internacional ISO 9001, traducción oficial. Sistemas de gestión de la calidad - Requisitos [quinta edición]. Suiza: Autor.

Socconini, L. (2017). Lean manufacturing paso a paso [primera edición]. México: Pandora.

Tapping, D. y Shuker, T. (2003). Value Stream Management for the Lean Office. Eight steps to planning, Mapping, and Sustaining Lean Improvements in Administrative areas. [Gestión de flujo de valor para la oficina esbelta. Ocho pasos para la planificación, mapeo y mantenimiento de mejoras esbeltas en áreas administrativas]. USA: Productivity Press.

Womack, J.; Jones, D. y Roos, D. (1990). The machine that changed the world: The story of lean production [La máquina que cambió al mundo: la historia de la producción esbelta] [primera edición]. New York: Rawson Associates. 